\title{
Endobronchial neurogenic tumor consisting of the features of a solitary circumscribed neuroma
}

\author{
Kazuya Tone ${ }^{1 \wedge}$, Takashi Yorozu ${ }^{2}$, Yuta Hirano ${ }^{1}$, Ryoko Sasaki ${ }^{1}$, Taisuke Kazuyori ${ }^{1}$, Akira Kojima ${ }^{1}$, \\ Kazuyoshi Kuwano ${ }^{3}$ \\ ${ }^{1}$ Department of Respiratory Medicine, The Jikei University School of Medicine, Katsushika Medical Center, Tokyo, Japan; ${ }^{2}$ Department of \\ Pathology, The Jikei University School of Medicine Katsushika Medical Center, Tokyo, Japan; ${ }^{3}$ Division of Respiratory Diseases, Department of \\ Internal Medicine, The Jikei University School of Medicine, Tokyo, Japan \\ Correspondence to: Kazuya Tone, MD, PhD. Division of Respiratory Diseases, Department of Internal Medicine, The Jikei University School of \\ Medicine, 3-25-8 Nishi-shimbashi, Minato-ku, Tokyo, Japan. Email: tone@jikei.ac.jp.
}

Submitted Jul 04, 2020. Accepted for publication Nov 15, 2020.

doi: $10.21037 /$ jtd-20-2365

View this article at: http://dx.doi.org/10.21037/jtd-20-2365

Solitary circumscribed neuroma $(\mathrm{SCN})$ is a benign neurogenic tumor that occurs in facial skin or oral mucosa (1). We encountered a case of a patient with an endobronchial neurogenic tumor with findings similar to those of SCN.

A 46-year-old Japanese man with a history of chronic renal failure on maintenance hemodialysis visited our hospital because of abnormal chest radiography findings at a periodic health checkup. He had no respiratory symptoms or particular histories of hereditary disease or trauma. Physical examination showed no abnormal vital signs or other findings. Chest computed tomography showed a protruding lesion on the left main bronchus (Figure 1A), and bronchoscopy revealed a polypoid tumor on the left main bronchus (Figure 1B). A biopsy forceps was used to resect the tumor. Histopathological examination revealed that the tumor had developed from the subepithelial bronchus with partial capsules. Spindle-shaped cells with eosinophilic cytoplasm and wavy nuclei had proliferated and showed a fascicular pattern (Figure 1C, hematoxylin and eosin staining). Cytological atypia was not present. The spindle cells were positive for S100 but negative for epithelial membrane antigen, smooth muscle actin, and desmin with immunostaining. The MIB-1 index was $2 \%$. A large number of neurofilament-positive axons were observed, and the clefts of the cell bundles were positive for CD34
(Figure 1C). Thus, we diagnosed him as having a benign endobronchial neurogenic tumor with features similar to those of SCN. The SCN was initially called a palisaded encapsulated neuroma (PEN), which was proposed by Reed et al. in 1972 as a benign peripheral nerve sheath tumor with unique pathological features different from those of other neurogenic tumors, such as schwannoma, neurofibroma, or traumatic neuroma $(2,3)$. Palisading arrangement or capsules are unclear in some PEN cases, so Fletcher et al. proposed using the name "SCN" instead of "PEN" because it properly reflects the pathological features (4). The majority of SCNs (90\%) occur in facial skin and oral or labial mucosa (5). Rarely, SCN occurs in the extremities or genital areas (6). However, to the best of our knowledge, no report has described an SCN that originated from the bronchial mucosa or lung. Some SCNs might be overlooked as a schwannoma or solitary mucosal neuroma because of insufficient immunostaining $(7,8)$. Nevertheless, $\mathrm{SCN}$ can be differentiated by using neurofilament staining (variably positive in SCN but not in schwannoma). A previous report demonstrated that multiple endocrine neoplasia type 2 (MEN2) should be differentiated because of similar pathological findings (9). However, our patient did not have a family history or physical findings associated with MEN2. Hence, we consider this to be the first case report of an endobronchial neurogenic tumor consisting of

\footnotetext{
$\wedge$ ORCID: 0000-0001-9898-4172.
} 

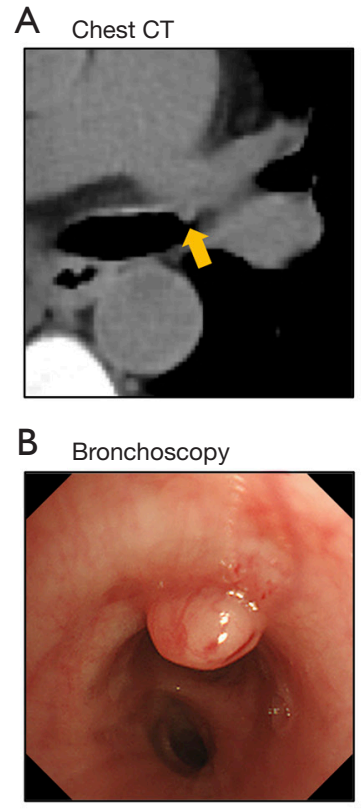

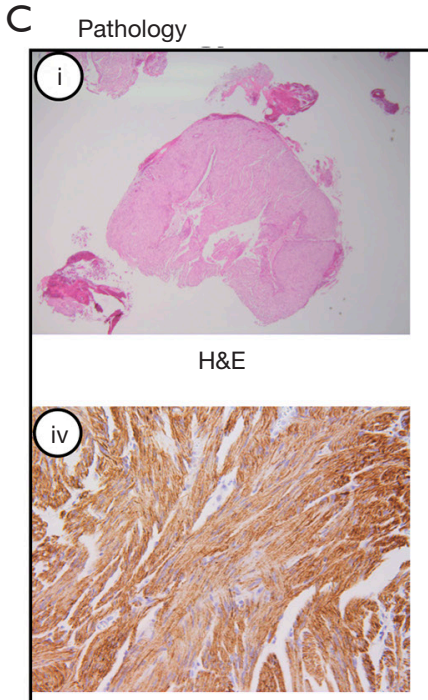

Neurofilament

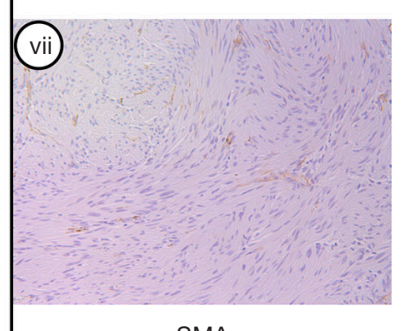

SMA

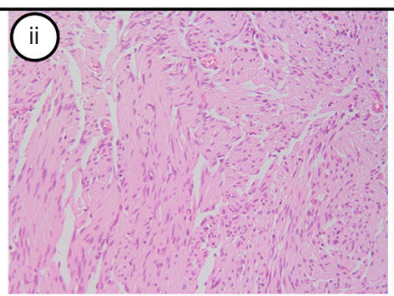

$H \& E$

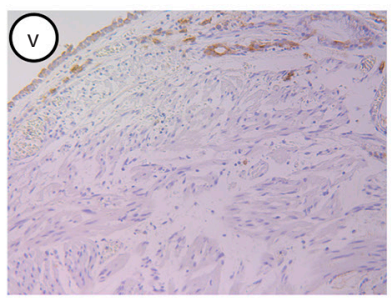

EMA

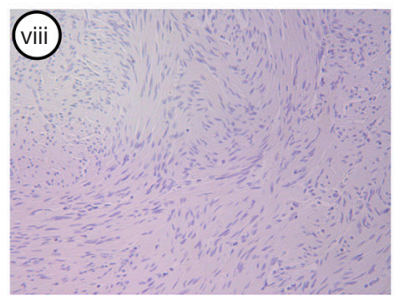

Desmin

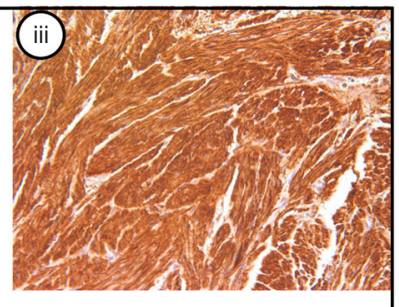

S100

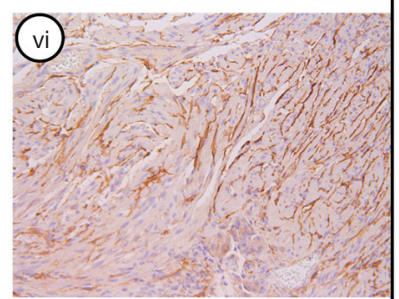

CD 34

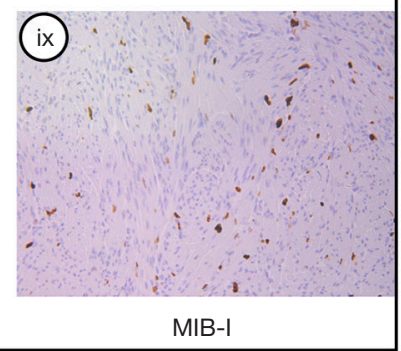

Figure 1 Laboratory findings. (A) Chest computed tomography showing a protruding lesion on the left main bronchus (arrow). (B) Bronchoscopic findings of the left main bronchus. (C). Pathological findings. (i) Hematoxylin and eosin (H\&E) staining ( $\times 20)$; (ii) H\&E staining ( $\times 200)$; (iii) S100 staining $(\times 200)$; (iv) neurofilament staining $(\times 200)$; (v) epithelial membrane antigen (EMA) staining $(\times 200)$; (vi) CD34 staining ( $\times 200)$; (vii) smooth muscle actin (SMA) staining ( $\times 200)$; (viii) desmin staining ( $\times 200)$; (ix) MIB-I staining ( $\times 200)$.

SCN features. Various types of immunostaining would help differentiate SCN from schwannoma or other neurogenic tumors, as in this case. Further studies are required to assess the prevalence and characteristics of endobronchial neurogenic tumors with the features of SCN.

\section{Acknowledgments}

We thank Dr. Akihiko Yoshida, Department of Pathology, National Cancer Center Hospital, for his helpful comments concerning the pathological findings.

Funding: None.

\section{Footnote}

Provenance and Peer Review: This article was a free submission to the journal. The article was sent for external peer review.

Conflicts of Interest: All authors have completed the ICMJE uniform disclosure form (available at http://dx.doi. org/10.21037/jtd-20-2365). The authors have no conflicts of interest to declare.

Ethical Statement: The authors are accountable for all aspects of the work in ensuring that questions related to the accuracy or integrity of any part of the work are appropriately investigated and resolved. Written informed consent was obtained from the patient.

Open Access Statement: This is an Open Access article distributed in accordance with the Creative Commons Attribution-NonCommercial-NoDerivs 4.0 International License (CC BY-NC-ND 4.0), which permits the non- 
commercial replication and distribution of the article with the strict proviso that no changes or edits are made and the original work is properly cited (including links to both the formal publication through the relevant DOI and the license). See: https://creativecommons.org/licenses/by-nc-nd/4.0/.

\section{References}

1. Koutlas IG, Scheithauer BW. Palisaded encapsulated ("solitary circumscribed") neuroma of the oral cavity: a review of 55 cases. Head Neck Pathol 2010;4:15-26.

2. Reed RJ, Fine RM, Meltzer HD. Palisaded, encapsulated neuromas of the skin. Arch Dermatol 1972;106:865-70.

3. Rossi G, Marchioni A, Agostini L, et al. Traumatic neuroma of the bronchi: bronchoscopy and histology of a hitherto unreported lesion. Am J Surg Pathol 2008;32:640-1.

4. Fletcher CD. Solitary circumscribed neuroma of the skin (so-called palisaded, encapsulated neuroma). A

Cite this article as: Tone K, Yorozu T, Hirano Y, Sasaki R, Kazuyori T, Kojima A, Kuwano K. Endobronchial neurogenic tumor consisting of the features of a solitary circumscribed neuroma. J Thorac Dis 2020;12(12):7498-7500. doi: 10.21037/jtd$20-2365$ clinicopathologic and immunohistochemical study. Am J Surg Pathol 1989;13:574-80.

5. Leblebici C, Savli TC, Yeni B, et al. Palisaded Encapsulated (Solitary Circumscribed) Neuroma: A Review of 30 Cases. Int J Surg Pathol 2019;27:506-14.

6. Navarro M, Vilata J, Requena C, et al. Palisaded encapsulated neuroma (solitary circumscribed neuroma) of the glans penis. Br J Dermatol 2000;142:1061-2.

7. Miura H, Kato H, Hayata $\mathrm{Y}$, et al. Solitary bronchial mucosal neuroma. Chest 1989;95:245-7.

8. Erdem I, Duman D, Eroglu S, et al. Endobronchial Mucosal Neuroma with Sarcoidosis. J Coll Physicians Surg Pak 2018;28:162-3.

9. Misago N, Toda S, Narisawa Y. The relationship between palisaded encapsulated neuroma and the mucocutaneous neuroma seen in multiple endocrine neoplasia $2 \mathrm{~b}$ syndrome: a histopathologic and immunohistochemical study. Am J Dermatopathol 2014;36:562-9. 